\title{
Aurora Kinase Inhibitor PF-03814735
}

National Cancer Institute

\section{Source}

National Cancer Institute. Aurora Kinase Inhibitor PF-03814735. NCI Thesaurus. Code C66946.

An aurora kinase inhibitor with potential antineoplastic activity. PF-03814735 binds to and inhibits aurora kinases, serine-threonine kinases that play essential roles in mitotic checkpoint control during mitosis. Inhibition of aurora kinases may result in an inhibition of cellular division and proliferation in tumor cells that overexpress aurora kinases. 